\title{
BMJ Open ATTIRE: Albumin To prevenT Infection in chronic liveR failurE: study protocol for a single-arm feasibility trial
}

To cite: China L, Muirhead N, Skene SS, et al. ATTIRE: Albumin To prevenT Infection in chronic liveR failurE: study protocol for a single-arm feasibility trial. BMJ Open 2016;6:e010132 doi:10.1136/bmjopen-2015010132

- Prepublication history and additional material is available. To view please visit the journal (http://dx.doi.org/ 10.1136/bmjopen-2015010132).

Received 6 October 2015 Revised 20 November 2015 Accepted 6 January 2016

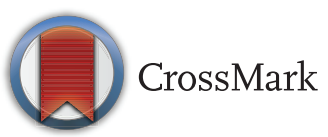

${ }^{1}$ Division of Medicine, University College London (UCL), London, UK ${ }^{2}$ Comprehensive Clinical Trials Unit, University College London (UCL), London, UK

Correspondence to

Louise China;

louise.china@ucl.ac.uk

\section{ABSTRACT}

Introduction: Circulating prostaglandin $\mathrm{E}_{2}$ levels are elevated in acutely decompensated cirrhosis and have been shown to contribute to immune suppression. Albumin binds and inactivates this hormone. Human albumin solution could thus be repurposed as an immune restorative drug in these patients.

This feasibility study aims to determine whether it is possible and safe to restore serum albumin to $>30 \mathrm{~g} / \mathrm{L}$ and maintain it at this level in patients admitted with acute decompensated cirrhosis using repeated $20 \%$ human albumin infusions according to daily serum albumin levels.

Methods and analysis: Albumin To prevenT Infection in chronic live R failurE (ATTIRE) stage 1 is a multicentre, open label dose feasibility trial. Patients with acutely decompensated cirrhosis admitted to hospital with a serum albumin of $<30 \mathrm{~g} / \mathrm{L}$ are eligible, subject to exclusion criteria. Daily intravenous human albumin solution will be infused, according to serum albumin levels, for up to 14 days or discharge in all patients. The primary end point is daily serum albumin levels for the duration of the treatment period and the secondary end point is plasma-induced macrophage dysfunction. The trial will recruit 80 patients. Outcomes will be used to assist with study design for an 866 patient randomised controlled trial at more than 30 sites across the UK.

Ethics and dissemination: Research ethics approval was given by the London-Brent research ethics committee (ref: 15/LO/0104). The clinical trials authorisation was issued by the medicines and healthcare products regulatory agency (ref: 20363/0350/ 001-0001).

Results: Will be disseminated through peer reviewed journals and international conferences. Recruitment of the first participant occurred on 26/05/2015.

Trial registration number: The trial is registered with the European Medicines Agency (EudraCT 2014-00230024) and has been adopted by the NIHR (ISRCTN 14174793). This manuscript refers to V.4.0 of the protocol; Pre-results.

\section{INTRODUCTION}

Liver disease is the only major cause of mortality currently increasing in the UK and is

\section{Strengths and limitations of this study}

- This study will demonstrate feasibility and safety of targeted albumin dosing in patients with acutely decompensated liver cirrhosis prior to a large randomised controlled trial (RCT).

- Biomarker end point provides validation of a novel biological assay for immune dysfunction.

- Outcomes will ensure optimal study protocol design for the RCT.

- Feasibility study therefore not randomised or powered to detect clinically relevant beneficial outcomes.

the fifth most common cause of death. ${ }^{1}$ These deaths are predicted to double over the next 20 years. ${ }^{2}$

Patients with symptoms of liver failure secondary to cirrhosis are described as acute decompensation (AD) patients. They are highly prone to bacterial infection ${ }^{3}$ secondary to immune dysfunction, ${ }^{4}$ with nosocomial (hospital-acquired) infection rates of $35 \%$ compared to $5 \%$ in non-cirrhotic patients. ${ }^{56}$ Of those that develop infection with organ dysfunction, 60-95\% die, often following prolonged intensive care unit (ICU) admission. ${ }^{7}$ There is, however, no medical strategy to restore immune competence.

It has been demonstrated that elevated circulating prostaglandin $\mathrm{E}_{2}\left(\mathrm{PGE}_{2}\right)$ levels contribute to immune suppression in $\mathrm{AD}$ patients. ${ }^{8}$ The plasma protein albumin binds and catalyses inactivation of $\mathrm{PGE}_{2}{ }^{9}$ Albumin is synthesised in the liver and levels fall as the synthetic function of the liver declines in advanced cirrhosis, making $\mathrm{PGE}_{2}$ more bioavailable. In addition the binding capacity of endogenous albumin is known to be defective in cirrhosis. ${ }^{10} 11$ We found a serum albumin of $<30 \mathrm{~g} / \mathrm{L}$ predicted plasma-induced macrophage dysfunction in a small cohort of $\mathrm{AD}$ patients ${ }^{8}$ and this was reversed when albumin levels were increased to $>30 \mathrm{~g} / \mathrm{L}$. 
We propose a novel strategy to repurpose $20 \%$ human albumin solution (HAS) as an immune restorative drug in $\mathrm{AD}$ patients with the aim of maintaining serum albumin at near normal levels.

Albumin To prevenT Infection in chronic liveR failurE (ATTIRE) incorporates a phase II single-arm multicentre feasibility trial $(\mathrm{n}=80)$ prior to a phase III randomised controlled trial (RCT) $(\mathrm{n}=866)$ assessing the impact of treatment on the incidence of nosocomial infections, organ dysfunction and mortality in patients admitted to hospital with $\mathrm{AD}$ of liver cirrhosis.

This feasibility trial aims to verify that daily intravenous human albumin infusions will restore serum albumin levels to near normal in $\mathrm{AD}$ patients, that this is safe and that there is physician equipoise prior to proceeding to a large RCT. Despite multiple studies, including systematic reviews, ${ }^{12}{ }^{13}$ evaluating albumin in septic intensive care patients there is a lack of interventional RCTs in patients with liver cirrhosis in which the mechanism of albumin's action is different. ${ }^{14-16}$ To date there has not been an albumin dosing trial aimed at increasing serum albumin levels in this context therefore it was essential that this was completed before proceeding to a large, interventional RCT. We shall also examine the effects on patient plasma-induced macrophage dysfunction using assays developed within our laboratory ${ }^{8}$ which will validate the proposed mechanism of albumin's action in patients with chronic liver failure.

\section{METHODS AND ANALYSIS}

\section{Primary objective}

To determine whether it is possible to restore to and maintain serum albumin at $>30 \mathrm{~g} / \mathrm{L}$ in patients admitted with $\mathrm{AD}$ using repeated 20\% HAS infusions according to daily measured serum albumin levels (figure 1).

\section{Secondary objective}

We shall assess patient plasma-induced macrophage dysfunction (as an indicator of immune suppression) in $\mathrm{AD}$ patients on the day of recruitment and during HAS treatment to determine whether this is substantially improved following albumin infusion. ${ }^{17}$

\section{Trial design}

This is a multicentre, open label single-arm feasibility trial in which all patients will be treated with $20 \%$ HAS to target levels above $30 \mathrm{~g} / \mathrm{L}$. Sequential patients admitted to $10 \mathrm{UK}$ participating hospitals with a clinical diagnosis of cirrhosis and $\mathrm{AD}$ will be screened using the inclusion and exclusion criteria (table 1).

\section{Clinical trial end points}

The primary end point is daily serum albumin level for the duration of the treatment period (maximum of 14 days or when the patient is considered fit for discharge if less than 14 days).

The key secondary end point is patient plasma-induced macrophage dysfunction assessed by our laboratory-based assays. ${ }^{8}$ Immune function is an extremely complex process for which there is no simple test or assay. During inflammation, monocytes move quickly to sites of tissue infection and differentiate into macrophages to elicit an immune response. Numerous studies have demonstrated the role of monocyte deactivation in cirrhosis associated immune suppression. ${ }^{18-20}$ It is, however, impractical to perform blinded, standardised, biological assays using fresh monocytes from 10 sites spread throughout the UK. As it has been demonstrated that circulating plasma mediators are responsible for monocyte and neutrophil dysfunction, ${ }^{21}{ }^{22}$ we developed an assay in which stored plasma from $\mathrm{AD}$ patients is added to macrophages from healthy donors. 8 This permits testing of patient samples from multiple sites at the same time in a controlled fashion.

We have selected macrophage production of the proinflammatory cytokine tumour necrosis factor $\alpha$ $(\mathrm{TNF}-\alpha)$ as our immune-readout as this has been validated as a biomarker of monocyte function in critical illness. Reduced capacity to produce TNF- $\alpha$ is associated with adverse outcomes following sepsis. ${ }^{23} 24$

We will stimulate human monocyte-derived macrophages with lipopolysaccharide (LPS) in the presence of $25 \%$ patient plasma pretreatment and post-treatment, measuring TNF- $\alpha$ production. LPS simulates a bacterial infectious stimulus. Improvement in macrophage function will be defined as an increase in LPS-induced TNF- $\alpha$ production. Plasma from healthy controls will also be used as a comparator.

Other secondary end points include rates of infection, organ dysfunction and mortality during the 14-day treatment period. Clinical data will also be collected regarding safety, type of infection, antibiotic prescribing, total amount of fluid administered, ICU admission and duration of hospital stay.

\section{Patient population}

This will include all patients admitted to hospital with complications of liver cirrhosis and serum albumin $<30 \mathrm{~g} / \mathrm{L}$, aged over 18 years with anticipated hospital length of stay of five or more days at trial enrolment, which should be no later than $72 \mathrm{~h}$ from admission. This is subject to exclusion criteria as detailed in table 1 . The diagnosis of cirrhosis will be performed by the clinical team as per standard UK practice and does not require liver biopsy or imaging.

\section{Consent}

Patient information sheets (see online supplementary appendix 1) will be given to and discussed with potential patients before consent is sought. Informed consent will be obtained from each participant or their legal representative. Patients who lack mental capacity, for any reason, are not excluded from the trial. An important subgroup of patients will have hepatic encephalopathy and these patients may lack capacity to consent. However, these patients may be among those that 
Figure 1 Protocol for ATTIRE phase II feasibility trial. ATTIRE, Albumin To prevenT Infection in chronic liveR failurE.

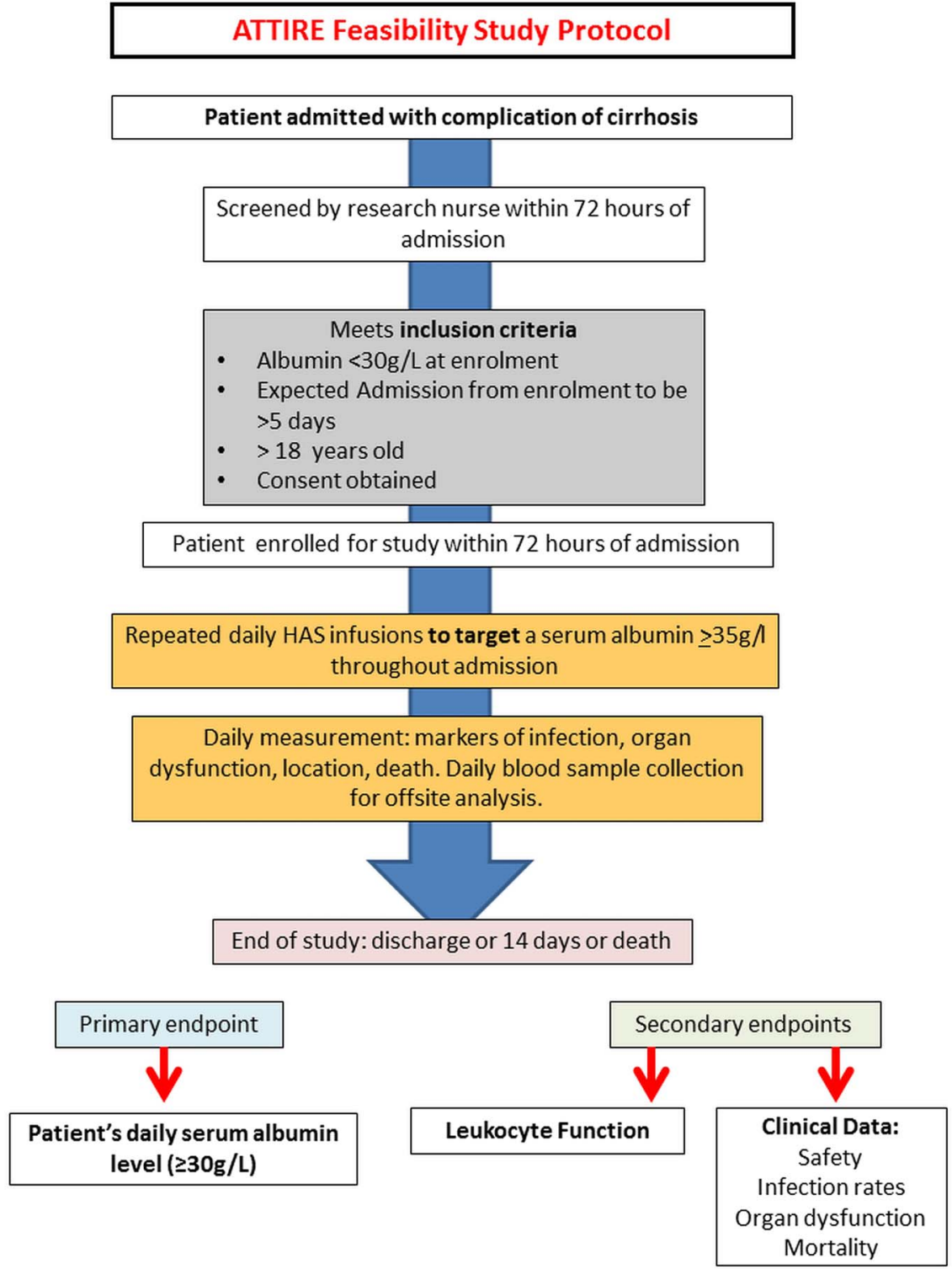

receive maximum benefit from the intervention. ${ }^{25-27}$ In this case consent will be sought from an appropriate legal representative independent of the research team as per current UK clinical trials regulations. ${ }^{28}$

\section{Intervention}

All patients will receive a daily infusion of $20 \%$ HAS intravenously $(100 \mathrm{~mL} / \mathrm{h})$ for a maximum of 14 days or until discharge (if less than 14 days). The volume of HAS prescribed each day will be determined by the patient's serum albumin level on that day.

Table 2 shows a suggested dosing protocol for albumin administration. This is based on the reported regimen used in the ALBIOS study ${ }^{29}$ and clinical experience as there are no prior albumin dose-increment studies in cirrhosis patients. In $\mathrm{ALBIOS}^{29}$ patients with a very low albumin $(<20 \mathrm{~g} / \mathrm{L})$ incremented to a higher value within 4-5 days therefore we would expect 20\% HAS requirements, as according to our trial protocol, to decrease after a few days with a subsequent decrease in cost and time of administration. However, there may be a subgroup of non-responders. We expect these patients to be very unwell and careful monitoring of side effects with on-going albumin infusion may warrant cessation of the trial intervention in this group. If this is the case additional time-to-increment advice may be added to the RCT protocol.

Differing regimens may be used to cover large volume paracentesis $(8 \mathrm{~g}$ of albumin/ $\mathrm{L}$ of ascites drained) or treat hepatorenal syndrome ( $1 \mathrm{~g}$ of albumin $/ \mathrm{kg}$ of body weight) as per international guidelines ${ }^{30} 31$ but HAS must be prescribed and given if serum albumin $<35 \mathrm{~g} / \mathrm{L}$. All variations will be recorded in the patient's daily Case Report Form (CRF).

\section{Evaluations during and after treatment}

Clinical, biochemical and microbiological data will be collected daily during the trial treatment period (see online supplementary appendix 2) using information from hospital notes that is recorded as standard of care. 
Table 1 Patient selection criteria

\begin{tabular}{|c|c|}
\hline Patient inclusion criteria & Patient exclusion criteria \\
\hline $\begin{array}{l}\text { All patients admitted to } \\
\text { hospital with acute onset or } \\
\text { worsening of complications } \\
\text { of cirrhosis }\end{array}$ & $\begin{array}{l}\text { Advanced hepatocellular } \\
\text { carcinoma with life } \\
\text { expectancy of less than } \\
8 \text { weeks }\end{array}$ \\
\hline Over 18 years of age & $\begin{array}{l}\text { Patients who will receive } \\
\text { palliative treatment only } \\
\text { during their hospital } \\
\text { admission }\end{array}$ \\
\hline $\begin{array}{l}\text { Predicted hospital } \\
\text { admission }>5 \text { days at trial } \\
\text { enrolment, which must be } \\
\text { within } 72 \mathrm{~h} \text { of admission }\end{array}$ & Patients who are pregnant \\
\hline $\begin{array}{l}\text { Serum albumin }<30 \mathrm{~g} / \mathrm{L} \text { at } \\
\text { screening }\end{array}$ & $\begin{array}{l}\text { Known or suspected severe } \\
\text { cardiac dysfunction }\end{array}$ \\
\hline \multirow[t]{3}{*}{$\begin{array}{l}\text { Documented informed } \\
\text { consent to participate (or } \\
\text { consent given by a legal } \\
\text { representative) }\end{array}$} & $\begin{array}{l}\text { Any clinical condition which } \\
\text { the investigator considers } \\
\text { would make the patient } \\
\text { unsuitable for the trial }\end{array}$ \\
\hline & $\begin{array}{l}\text { The patient has been } \\
\text { involved in a clinical trial of } \\
\text { Investigational Medicinal } \\
\text { Products (IMPs) within the } \\
\text { previous } 30 \text { days that would } \\
\text { impact on their participation } \\
\text { in this study }\end{array}$ \\
\hline & $\begin{array}{l}\text { Trial investigators unable to } \\
\text { identify the patient (by NHS } \\
\text { number) }\end{array}$ \\
\hline
\end{tabular}

NHS, National Health Service.

There is no follow-up beyond the treatment period. The blood samples collected for immune function tests will be analysed in a blinded fashion at a central site.

\section{Statistical considerations}

Sample size

The primary purpose of this Feasibility Trial is to demonstrate that repeated 20\% HAS infusions can raise and maintain serum albumin at $\geq 30 \mathrm{~g} / \mathrm{L}$ in liver cirrhosis patients presenting with $\mathrm{AD}$.

Table 2 Dosing protocol for 20\% HAS administration (amounts per day) as advised by measured serum albumin level on that day

\begin{tabular}{ll}
\hline $\begin{array}{l}\text { Patient's serum albumin } \\
\text { level (g/L) }\end{array}$ & $\begin{array}{l}\text { Amount of } \mathbf{2 0} \% \\
\text { HAS to be administered } \\
(\mathbf{m L})\end{array}$ \\
\hline$\geq 35$ & None \\
$30-34$ & 100 \\
$26-29$ & 200 \\
$20-25$ & 300 \\
$<20$ & 400 \\
\hline
\end{tabular}

HAS, human albumin solution.
Eighty patients will be recruited. Success would be demonstrated if $60 \%$ of patients were able to achieve and maintain serum albumin levels at or above $30 \mathrm{~g} / \mathrm{L}$ on at least $1 / 3$ of days in which the level is recorded. We believe that there will be a subgroup of patients with very low albumin levels who may not be able to achieve this end point which has influenced our definition of success. If our assumptions are correct these patients will be excluded from RCT recruitment. With 72 evaluable patients (allowing for $10 \%$ loss-to-follow-up/withdrawal) the probability of achieving 44 or more 'successes' is around $80 \%$ assuming that each patient has a $65 \%$ chance of attaining the required level.

The trial will be performed at 10 sites. 8-10 patients per site will allow identification of any variability in the delivery of the albumin-targeting dose protocol between centres. It will be compulsory to record reason for protocol variation in the daily CRF.

\section{Statistical evaluation}

As this is a feasibility trial the emphasis will be on producing relevant data summaries rather than on formal modelling or hypothesis testing. This will support the IDMC and TSC in deciding whether to recommend proceeding with the RCT.

\section{Primary outcome}

Serum albumin levels will be summarised for each of days $1-15$ by table, mean \pm SD and graph (median level vs day with superimposed bars displaying IQR). The numbers of patients observed on each day will be noted. Day 1 will represent the baseline serum albumin level before the first administration of 20\% HAS according to the protocol.

The number of patients on each day whose serum albumin level exceeds $30 \mathrm{~g} / \mathrm{L}$ will be noted as a percentage of those evaluated, together with the overall percentage of patients whose serum albumin level exceeds $30 \mathrm{~g} / \mathrm{L}$ on at least $1 / 3$ of the days on which it is recorded.

\section{Secondary outcomes}

Albumin's ex vivo impact on immune function will be determined by comparing macrophage function in the presence of patient plasma using our laboratory assays when their albumin level is $<30 \mathrm{~g} / \mathrm{L}$ compared to $\geq 30 \mathrm{~g} / \mathrm{L}$. A substantial improvement in macrophage function is expected following treatment with albumin.

Information shall be summarised regarding the total volume of albumin infused and the total amount of fluid administered, days in ICU and duration of hospital stay, together with the rates of nosocomial infections, organ dysfunction and in-hospital mortality which are the component elements of the composite end point for the RCT. Safety will be assessed by consideration of the number of SAEs reported during the trial.

Data will be further summarised within 'groups' defined by baseline serum albumin levels $(<20,20-25$ and $26-29 \mathrm{~g} / \mathrm{L}$ ) to investigate whether there are any 
apparent differences in outcome by group. This subgroup analysis is exploratory, since we are not powered to detect differences, but may be useful in identifying whether any amendments are necessary to the protocol for the RCT.

\section{DISCUSSION}

ATTIRE is a UK multicentre trial that aims to evaluate the repurposing of HAS as an immune restorative drug. This protocol describes the feasibility trial which will determine if it is possible and safe to raise and maintain $\mathrm{AD}$ patients' serum albumin levels to $>30 \mathrm{~g} / \mathrm{L}$.

In liver cirrhosis current evidence-based guidance ${ }^{31-33}$ advocates the use of HAS in large volume paracentesis, ${ }^{34}$ hepatorenal syndrome ${ }^{35}$ and spontaneous bacterial peritonitis. ${ }^{36} 37$ To date there has not been an albumin dosing trial aimed at increasing serum albumin levels. Therefore it was essential that this study was completed before proceeding to a large, interventional RCT.

Studies evaluating the safety of HAS infusions have generally shown it to be a safe treatment. ${ }^{12} 132934$ The main concerns in the cirrhotic population are related to volume overload leading to pulmonary oedema and increase in portal pressure leading to variceal bleeding. A recent interventional trial in septic $\mathrm{AD}$ patients reported an $8.3 \%$ rate of pulmonary oedema in the albumin treatment group. ${ }^{38}$ However, the weight based albumin dosing regimen in this study led to much larger daily volumes of albumin being prescribed than suggested in our protocol. This and other studies in cirrhosis have not reported an increased incidence in variceal bleeding. ${ }^{34} 3739$

We shall also correlate our primary end point of a numerical increase in albumin levels with a biological outcome measuring markers of immune function ex vivo. This aims to verify that increasing serum albumin above $30 \mathrm{~g} / \mathrm{L}$ is associated with an improvement in patient plasma-induced macrophage dysfunction ex vivo in a much larger patient cohort than already shown. ${ }^{8}$ These outcomes will be used to move forward and if appropriate modify the protocol for the ATTIRE RCT.

\section{Ethics and dissemination}

This research group will involve a potentially vulnerable patient group that have hepatic encephalopathy and therefore lack the capacity to consent. However, patients with encephalopathy are at high risk of infection and could be those that potentially receive maximal benefit from the intervention and therefore should not be denied access to the trial treatment. We have undertaken steps to ensure these patients are appropriately recruited to the trial (described in 'Consent' section) and provided individual site training.

Research Ethics positive opinion was given by the London-Brent Research Ethics Committee (ref: 15/LO/ 0104) which specialise in trials involving patients who lack the capacity to consent.
The Clinical Trials Authorisation was issued by the Medicines and Healthcare products Regulatory Agency (MHRA, ref: 20363/0350/001-0001). The trial is registered with the European Medicines Agency (EudraCT 2014-002300-24) and has been adopted by the NIHR. Recruitment started in May 2015 and will finish by November 2015. Assuming feasibility milestones have been met a 866 patient phase III randomised control trial (ATTIRE stage 2) will start at the end of 2015 randomising patients to daily HAS infusion or routine standard of care.

Study findings will be disseminated through peer reviewed publications and international conference presentations. They will also be used to generate the study protocol for a large interventional RCT (ATTIRE stage 2).

\section{Trial funding and sponsor}

The work is supported by the Health Innovation Challenge fund (Wellcome Trust and Department of Health) award number 164699. The trial sponsor is UCL with trial management activities conducted by the UCL Comprehensive Clinical Trials Unit.

\section{TRIAL MANAGEMENT AND MONITORING Research Steering Group}

The Research Steering Group (RSG) operates on behalf of the funders to ensure that appropriate milestones have been met in the delivery of the trial. It consists the CI, an independent expert and representatives of the Welcome Trust and Department of Health.

\section{Trial Management Group}

The Trial Management Group (TMG) comprises the CI, Clinical Research Fellow, Clinical Project Manager, Trial Statistician, Trial Manager, Data Manager, Health Economist and five trial site PIs. The TMG is responsible for developing the design, co-ordination and strategic management of the trial.

\section{Trial Steering Committee}

The Trial Steering Committee (TSC) is the independent group responsible for oversight of the trial in order to safeguard the interests of trial patients. The TSC provides advice to the CI, Clinical Trials Unit (CTU), funder and sponsor on all aspects of the trial through its independent chair.

\section{Independent Data Monitoring Committee}

The Independent Data Monitoring Committee (IDMC) is responsible for safeguarding the interests of trial patients, monitoring the accumulating data and making recommendations to the TSC on whether the trial should continue as planned. It comprises a clinical chair (independent hepatologist), independent gastroenterologist and an independent statistician all with expertise in Clinical Trials. 
Acknowledgements The authors have had the support of the following individuals via trial oversight committees: Professor Graeme Alexander, Professor Stephen Brett, Professor Mauro Bernardi, Professor Dominique Valla, Dr Vipul Jaraith, Mr Tim Clayton, Mr Brennan Kahan, Dr James O'Beirne, Dr Jim Portal, Dr Gavin Wright, Dr Ewan Forrest, Dr Stephen Ryder, Mr John Crookenden, Ms Susan Tebbs, A list of trial sites and Pls can be obtained by contacting attire@ucl.ac.uk.

Contributors All authors read and approved the final manuscript. LC was involved in the protocol development, writing of this manuscript; NM was involved in the protocol development, manuscript review; ZS was involved in the protocol development, manuscript review; SS was involved in the protocol development, statistical input, manuscript review; AM: manuscript review, design of laboratory analysis; DG: manuscript review, design of laboratory analysis; A0 was involved in the concept and design, protocol development, writing of this manuscript.

Funding Health Innovation Challenge fund (Wellcome Trust and Department of Health) (grant no. 164699)

Competing interests None declared.

Ethics approval London-Brent Research Ethics Committee (ref: 15/L0/0104).

Provenance and peer review Not commissioned; externally peer reviewed.

Open Access This is an Open Access article distributed in accordance with the terms of the Creative Commons Attribution (CC BY 4.0) license, which permits others to distribute, remix, adapt and build upon this work, for commercial use, provided the original work is properly cited. See: http:// creativecommons.org/licenses/by/4.0/

\section{REFERENCES}

1. Health Service Quarterly. Vol. No. 40 (ed. Statistics, O.f.N.) p59-60 (Winter 2008)

2. Williams $\mathrm{R}$, Aspinall $\mathrm{R}$, Bellis $\mathrm{M}$, et al. Addressing liver disease in the UK: a blueprint for attaining excellence in health care and reducing premature mortality from lifestyle issues of excess consumption of alcohol, obesity, and viral hepatitis. Lancet 2014;384:1953-97.

3. Fernández J, Navasa M, Gómez J, et al. Bacterial infections in cirrhosis: epidemiological changes with invasive procedures and norfloxacin prophylaxis. Hepatology 2002;35:140-8.

4. Rajkovic I, Williams R. Abnormalities of neutrophil phagocytosis, intracellular killing and metabolic activity in alcoholic cirrhosis and hepatitis. Hepatology 1986;6:252-62.

5. Acevedo J, Fernández J. New determinants of prognosis in bacterial infections in cirrhosis. World J Gastroenterol 2014;20:7252-9.

6. Fernández $\mathrm{J}$, Acevedo $\mathrm{J}$, Castro $\mathrm{M}$, et al. Prevalence and risk factors of infections by multiresistant bacteria in cirrhosis: a prospective study. Hepatology 2012;55:1551-61.

7. O'Brien AJ, Welch CA, Singer M, et al. Prevalence and outcome of cirrhosis patients admitted to UK intensive care: a comparison against dialysis-dependent chronic renal failure patients. Intensive Care Med 2012;38:991-1000.

8. O'Brien AJ, Fullerton JN, Massey KA, et al. Immunosuppression in acutely decompensated cirrhosis is mediated by prostaglandin E2. Nat Med 2014:20:518-23.

9. Yang J, Petersen CE, $\mathrm{Ha} \mathrm{CE}$, et al. Structural insights into human serum albumin-mediated prostaglandin catalysis. Protein $\mathrm{Sci}$ 2002;11:538-45.

10. Klammt S, Mitzner S, Stange J, et al. Albumin-binding function is reduced in patients with decompensated cirrhosis and correlates inversely with severity of liver disease assessed by model for end-stage liver disease. Eur J Gastroenterol Hepatol 2007:19:257-63.

11. Jalan R, Schnurr K, Mookerjee RP, et al. Alterations in the functional capacity of albumin in patients with decompensated cirrhosis is associated with increased mortality. Hepatology 2009;50:555-64.

12. Patel A, Laffan MA, Waheed U, et al. Randomised trials of human albumin for adults with sepsis: systematic review and meta-analysis with trial sequential analysis of all-cause mortality. BMJ 2014;349: g4561.

13. Cochrane Injuries Group Albumin Reviewers. Human albumin administration in critically ill patients: systematic review of randomised controlled trials. BMJ 1998;317:235-40.

14. Arroyo V, García-Martinez R, Salvatella X. Human serum albumin, systemic inflammation, and cirrhosis. J Hepatol 2014;61:396-407.
15. Domenicali M, Baldassarre M, Giannone FA, et al Posttranscriptional changes of serum albumin: clinical and prognostic significance in hospitalized patients with cirrhosis. Hepatology 2014;60:1851-60.

16. Garcia-Martinez R, Andreola F, Mehta G, et al. Immunomodulatory and antioxidant function of albumin stabilises the endothelium and improves survival in a rodent model of chronic liver failure. $J$ Hepatol 2015;62:799-806.

17. Venet F, Lukaszewicz AC, Payen D, et al. Monitoring the immune response in sepsis: a rational approach to administration of immunoadjuvant therapies. Curr Opin Immunol 2013;25:477-83.

18. Wasmuth HE, Kunz D, Yagmur E, et al. Patients with acute on chronic liver failure display "sepsis-like" immune paralysis. J Hepatol 2005;42:195-201.

19. Antoniades CG, Wendon J, Vergani D. Paralysed monocytes in acute on chronic liver disease. J Hepatol 2005;42:163-5.

20. Xing $\mathrm{T}, \mathrm{Li} \mathrm{L}, \mathrm{CaO} \mathrm{H}$, et al. Altered immune function of monocytes in different stages of patients with acute on chronic liver failure. Clin Exp Immunol 2007;147:184-8.

21. Lin CY, Tsai IF, Ho YP, et al. Endotoxemia contributes to the immune paralysis in patients with cirrhosis. $J$ Hepatol 2007;46:816-26.

22. Mookerjee RP, Stadlbauer V, Lidder S, et al. Neutrophil dysfunction in alcoholic hepatitis superimposed on cirrhosis is reversible and predicts the outcome. Hepatology 2007;46:831-40.

23. Docke WD, Randow F, Syrbe U, et al. Monocyte deactivation in septic patients: restoration by IFN- $\gamma$ treatment. Nat Med 1997;3:678-81.

24. Ploder M, Pelinka L, Schmuckenschlager C, et al. Lipopolysaccharide-induced tumor necrosis factor alpha production and not monocyte human leukocyte antigen-DR expression is correlated with survival in septic trauma patients. Shock 2006;25:129-34.

25. Bajaj JS, O'Leary JG, Reddy KR, et al. Survival in infection-related acute-on-chronic liver failure is defined by extrahepatic organ failures. Hepatology 2014;60:250-6.

26. Bajaj JS, Ridlon JM, Hylemon PB, et al. Linkage of gut microbiome with cognition in hepatic encephalopathy. Am J Physiol Gastrointest Liver Physiol 2012;302:G168-175.

27. Simón-Talero M, García-Martínez R, Torrens M, et al. Effects of intravenous albumin in patients with cirrhosis and episodic hepatic encephalopathy: a randomized double-blind study. J Hepatol 2013;59:1184-92.

28. Medicines for Human Use (Clinical Trials Regulations) 2004 Informed consent in clinical trials. (ed. Authority, H.R.). 2008. http:// www.hra.nhs.uk/

29. Caironi P, Tognoni G, Masson S, et al. Albumin replacement in patients with severe sepsis or septic shock. N Engl J Med 2014;370:1412-21.

30. Angeli $P$, Ginès $P$, Wong $F$, et al. Diagnosis and management of acute kidney injury in patients with cirrhosis: revised consensus recommendations of the International Club of Ascites. $J$ Hepatol 2015;62:968-74.

31. European Association for the Study of the Liver. EASL clinical practice guidelines on the management of ascites, spontaneous bacterial peritonitis, and hepatorenal syndrome in cirrhosis. $J$ Hepatol 2010;53:397-417.

32. Moore KP, Aithal GP. Guidelines on the management of ascites in cirrhosis. Gut 2006:55(Suppl 6):vi1-12.

33. Runyon BA, AASLD. Introduction to the revised American association for the study of liver diseases practice guideline management of adult patients with ascites due to cirrhosis 2012 Hepatology 2013;57:1651-3

34. Bernardi M, Caraceni P, Navickis RJ, et al. Albumin infusion in patients undergoing large-volume paracentesis: a meta-analysis of randomized trials. Hepatology 2012;55:1172-81.

35. Martin-Llahi M, Pépin MN, Guevara M, et al. Terlipressin and albumin vs albumin in patients with cirrhosis and hepatorenal syndrome: a randomized study. Gastroenterology 2008;134:1352-9.

36. Fernández J, Navasa M, Garcia-Pagan JC, et al. Effect of intravenous albumin on systemic and hepatic hemodynamics and vasoactive neurohormonal systems in patients with cirrhosis and spontaneous bacterial peritonitis. J Hepatol 2004;41:384-90.

37. Sort $P$, Navasa M, Arroyo V, et al. Effect of intravenous albumin on renal impairment and mortality in patients with cirrhosis and spontaneous bacterial peritonitis. N Engl J Med 1999;341:403-9.

38. Thévenot $\mathrm{T}$, Bureau $\mathrm{C}$, Oberti $\mathrm{F}$, et al. Effect of albumin in cirrhotic patients with infection other than spontaneous bacterial peritonitis. A randomized trial. J Hepatol 2015;62:822-30.

39. Guevara M, Terra C, Nazar A, et al. Albumin for bacterial infections other than spontaneous bacterial peritonitis in cirrhosis. A randomized, controlled study. J Hepatol 2012;57:759-65. 